\title{
M-Geocast: Robust and Energy-Efficient Geometric Routing for Mobile Sensor Networks*
}

\author{
Lynn Choi ${ }^{1}$, Jae Kyun Jung ${ }^{1}$, Byong-Ha Cho ${ }^{1}$, and Hyohyun Choi ${ }^{2}$ \\ ${ }^{1}$ Korea University, Anam-Dong, Sungbuk-Ku, Seoul, Korea \\ \{lchoi, kernel, sntblue\} @korea.ac.kr \\ Tel.: +82-2-3290-3249; Fax: +82-2-921-0544 \\ ${ }^{2}$ u-Convergence Laboratory, Samsung Electronics Co., Ltd \\ hyohyun. choi@samsung.com
}

\begin{abstract}
In this paper we investigate a practical routing solution for a new class of wireless sensor networks where any node can be mobile anytime. Assuming GPS-enabled sensor nodes we propose a new geometric routing protocol called M-Geocast that is designed to efficiently support node mobility as well as location service for such mobile sensor networks. Unlike existing geometric routing schemes, M-Geocast can also discover a non-geometric path to the destination by exploiting the path history of location updates. Thus, the routing void can be minimized by alternating the two. In addition, M-Geocast employs two location-based optimizations to further reduce the overhead of ondemand route discovery on inevitable routing voids. Through detailed NS-2 simulations we demonstrate that M-Geocast can not only increase network performance but can also reduce energy consumption compared to the existing protocols based on on-demand route discovery or a plain geometric routing.
\end{abstract}

Keywords: mobility, sensor networks, geometric routing, MANET, void.

\section{Introduction}

Sensors are now sufficiently small and cheap so that people can carry them around. Coupling these sensors with a nearly billion mobile wireless devices will create a new network of mobile sensors in the future. Yet, most of existing studies on wireless sensor networks assume only stationary sensor nodes [1]. Recently several studies investigate the use of mobile sinks to improve coverage, localization accuracy, or energy efficiency $[2,4,9,11]$. However, none of these studies have investigated the case of fully mobile sensor network (MSN) environment where any node can move anytime. In this paper we investigate a practical routing solution for this new class of wireless sensor networks where both sensor nodes and sinks can be mobile anytime.

Node mobility brings several challenges to large-scale sensor networking. First, the preconstruction of message delivery network may not be useful since the topology may change too frequently due to node movement. Second, the frequent location updates

* This work was supported by the Korea Science and Engineering Foundation (KOSEF) grant funded by the Korea government (MOST) (No. R01-2007-000-20958-0). This work was also supported by a Korea University Grant. 
from a mobile node can lead to an excessive drain of sensors' limited battery power and increased collisions in wireless transmissions. Third, the situation can get worse when the number of mobile nodes grows. While MANET protocols [3, 8] may provide a reasonable performance for MSN but they would incur too much overhead in terms of traffic and energy consumption since they usually assume peer-to-peer randomized traffic rather than many-to-one traffic from sources to sinks assumed in MSN. One possible solution is employing a geometric routing scheme $[5,6,7]$ rather than a topology-based routing protocol. Assuming location-aware sensor nodes the geometric routing is scalable, fast, and energy efficient since it does neither require global routing table maintenance nor the on-demand path discovery used in MANET protocols.

In this paper, we propose a new geometric routing protocol called M-Geocast to efficiently support routing for mobile sensor networks. In the presence of multiple sinks M-Geocast designates one of the sinks as the master sink. The master sink acts as a location service provider and also as a data collection and dissemination server. Only the master sink needs to flood its location information. Thus, all the nodes including sinks can send messages to the master sink using a simple geographic forwarding. As in all the geometric schemes, M-Geocast may encounter geographic holes $[5,6,7]$. To recover from such a routing void, M-Geocast uses the following novel approach. First, M-Geocast can discover a second path to the destination by using the path history of the location updates. This is called path history forwarding. Second, if both the geometric routing and path history forwarding fail either due to a routing hole or due to a broken link, M-Geocast can discover a new path to the destination on demand by flooding RREQ messages. Thus, M-Geocast can completely eliminate the routing void. To avoid excessive flooding caused by such on-demand path discovery, M-Geocast employs two location-based optimization techniques called path history projection and geographic void prediction. Together, M-Geocast can substantially outperform existing routing solutions in terms of both energy efficiency and network performance as demonstrated in Section IV.

The rest of this paper is organized as follows. Section II surveys the related work. Section III introduces M-Geocast's routing, location service, and detour methods. Section IV discusses the simulation results. Finally, Section VI concludes the paper.

\section{Related Works}

Numerous geometric routing schemes $[5,6,7]$ have been proposed for ad hoc networks. In terms of forwarding strategy, existing greedy routing schemes can be classified as Greedy (minimize the distance to the destination) MFR (most forward progress within radius r), NFP (nearest with forward progress), Compass routing (select a neighbor closest to the straight line between the source and the destination), and Random selection depending on which neighbor node to forward to [7]. M-Geocast can be classified as Greedy, which selects a neighbor node that is closest to the destination as the next forwarding node.

In geometric routing a location service is necessary to learn the current position of a potential destination. Mobile nodes should register their current position with the location service provider. GLS [6] partitions the network field as a hierarchy of squares called quadtree. By issuing position queries up in the hierarchy, GLS ensures 
the position query to reach the correct location server. Another strategy called Homezone [10] uses a hash function to locate the server. Both GLS and Homezone can be classified as an all-for-some service [7] since all nodes need to store the location information for some other nodes. In terms of location service, M-Geocast can be classified as some-for-some approach [7], since only a single location server maintains the position information of mobile sinks.

In geometric greedy forwarding any node may fail to find the next node when it arrives at a local maximum where none of its neighbors is closer to the destination than itself. To counter this problem, numerous schemes have been proposed $[5,7]$. One intuitive strategy is selecting the node with the least backward progress if no nodes can be found in the forward direction. However, this leads to a looping problem [7]. The face-2 algorithm [7] and the perimeter routing strategy of GPSR [5] uses a simple planar graph traversal to find a path to the destination. However, this approach may fail to find a path when the network field has an open void [7]. In contrast, M-Geocast uses path history and other hints to recover from the routing holes and can guarantee the recovery by using the on-demand route discovery if necessary.

The idea of master sink is similar to the virtual sink concept used in VSR [2] which can support sink mobility assuming stationary sensor nodes. However, M-Geocast is different from VSR in the following aspects. First, M-Geocast does neither maintain routing table nor routing tree as in VSR. Second, VSR assumes only stationary sensor nodes. To support MSN, VSR needs to rebuild its routing tree continuously whenever there is a topology change, which might be too expensive to be used in MSN. Third, only a sink can perform the role of the master sink in M-Geocast, while a normal stationary sensor node is designated as a virtual sink in VSR. In summary, M-Geocast is a position-based routing protocol for mobile sensor networks while VSR is a topology-based routing protocol for static sensor networks with mobile sinks.

\section{M-Geocast}

\subsection{M-Geocast and Master Sink}

As in all geographical routing, M-Geocast marks each packet with the location information of its destination. A forwarding node can make a locally optimal, greedy choice by selecting one of its neighbors that is closest to the destination. This assumes that each node has the location information of all of its neighbors through neighbor discovery process as explained in Section III.2. In the presence of multiple mobile sinks M-Geocast designates one of the sinks as the master sink. The master sink acts as a data collection and dissemination server for all the nodes in the field. To accomplish this task, the master sink needs to periodically flood its location information throughout the sensor field. Thus, all the nodes in the field can send messages to the master sink using the geographic greedy forwarding. Figure 1 shows the routing path of M-Geocast when there are three sources and two destinations. Each source directs its messages to the master sink using the greedy forwarding. The master sink collects all the messages from the sources and forwards them to other sinks. To accomplish this, the master sink needs to track the location of other sinks. 


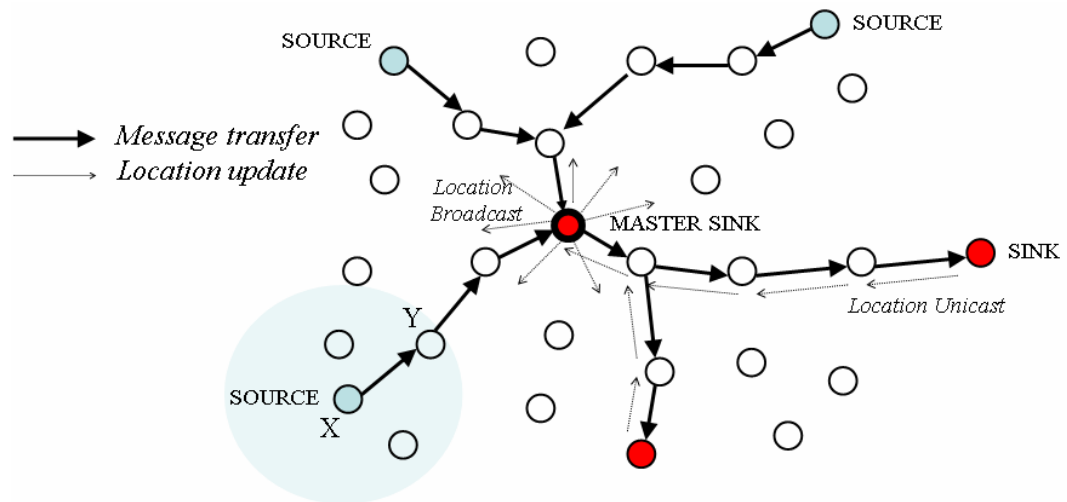

Fig. 1. Routing through a master sink in M-Geocast

\subsection{Location Service and Neighbor Discovery}

Adaptive Beaconing: For the geographic forwarding, each node in M-Geocast should maintain the location information of its neighbors. For this purpose, each node periodically broadcasts its location information to its neighbors. A simple beaconing provides all nodes with their neighbors' positions. Periodically, each node transmits a beacon using a MAC-level broadcast including only its own identifier (e.g., IP address) and position. However, in random waypoint model each node spends a significant portion of its time without movement. In stationary state, the periodic broadcast may generate too much unnecessary traffic. To avoid this, M-Geocast requires each node to broadcast its location only during movement. However, this creates another problem. Assume that a new node $\mathrm{X}$ moves in the area of node $\mathrm{Y}$ and node $\mathrm{Y}$ is in stationary state. Since node Y does not broadcast its location, the new node X cannot recognize the node $\mathrm{X}$ even though it does exist. To solve this problem, we propose a new neighbor discovery scheme called adaptive beaconing. First, a stationary node usually does not broadcast its beacon. However, whenever it hears the beacon of a new incoming node, it generates its beacon just once to inform its location to this newcomer. This allows each node to keep track of their neighbors' locations even during movement while suppressing the unnecessary beacon transmissions.

Location Broadcast from Master Sink: While the location information of a sensor node needs to be broadcast among its own neighbors, the location information of a master sink needs to be propagated throughout the sensor field because all the nodes assume the location information of the master sink. This is accomplished by a simple periodic flooding by the master sink. As in adaptive beaconing, the master sink needs to flood its location information only during its movement.

Location Unicast from Sink: To forward all the messages collected from sensor nodes, the location information of other sinks must be tracked by the master sink. This is accomplished by a simple unicast from each sink to the master sink by using the geometric greedy forwarding. Like in other location updates, this location update 
is needed only during the sink movement. Note that without the master sink the location service cannot be provided by such a simple unicast.

\subsection{Detour Methods for Routing Voids}

The power of greedy forwarding comes with one attendant drawback: there are topologies in which the only route to a destination requires a packet move temporarily farther in geometric distance from the destination [5]. A simple example of such a topology is shown in Figure 2. Here, a forwarding node $\mathrm{X}$ is closer to the master sink than all of its neighbors. The greedy forwarding cannot make further progress. This kind of routing void is called a geographic hole. To recover from this situation, we propose two different detour strategies: detouring with path history and detouring with on-demand route discovery.

Detour with Path History: The idea of this detour method is to use the path history of location updates. Note that the path from a source to a sink follows a reverse path of location updates from the sink to the source via the master sink. Figure 2 illustrates routing holes and a detour path from the source to the real sinks. It takes advantage of the fact that every node receives the location update from the master sink. If each node marks the neighbor node from which it has received the last location update, then it can follow the reverse path of the location update to find a path to the master sink as well as a path to the other sinks. In fact, M-Geocast can use two different routing strategies: one based on geometric greedy forwarding and the other based on the path history. We will investigate the performance impact of combining two different routing strategies in Section IV.

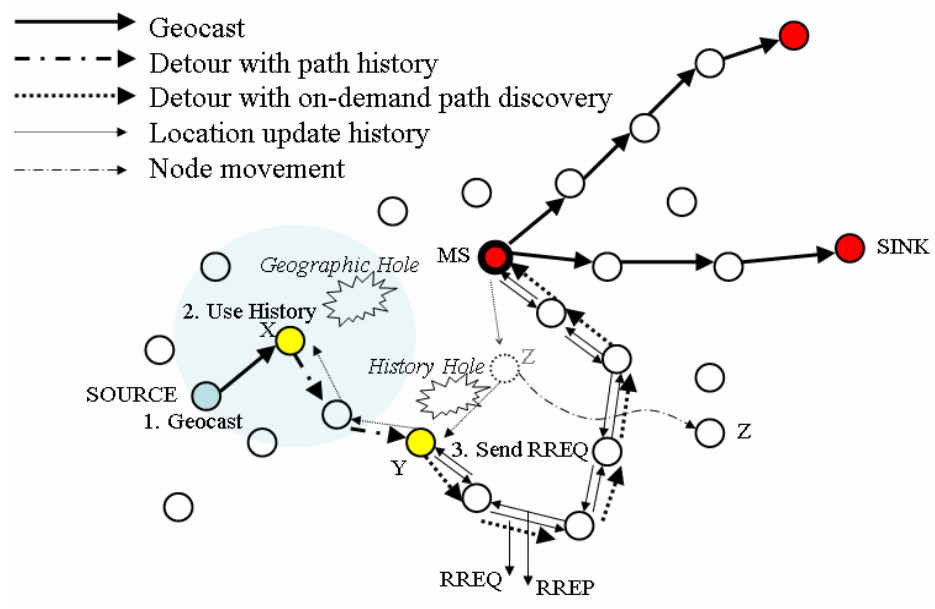

Fig. 2. Detour mechanisms of M-Geocast

Detour with On-Demand Route Discovery: The path history remains valid as long as the all the nodes in the path remain stationary. However, as time goes by, it is likely that a link in the path is broken due to node movement. The probability of the 
path failure increases as the distance between the source and the destination increases. We call this type of routing void as a history hole. For example, node $\mathrm{Y}$ in Figure 2 has a broken link to node $\mathrm{Z}$ due to Z's movement. If both the geometric routing and the path history routing lead to a routing void, we must resort to another detour method. Although several detour methods have been proposed [5, 7], theoretically it is possible to come up with a topology that can invalidate any sophisticated detour method based on a non-exhaustive search. To completely eliminate the routing holes, we use on-demand path discovery used in reactive MANET routing protocols [3, 8].

On a routing void, the forwarding node broadcasts RREQ (Route Request) which includes the address of itself, the destination address, and message type (RREQ). The destination node that receives RREQ replies with RREP (Route Reply) unicast including its location information. However, unlike AODV [8], M-Geocast does neither use source sequence numbers nor it does set up reverse route entries since it assumes unidirectional traffic from sources to sinks. The RREQ/RREP method is a robust detour method as it always finds a path to the destination as long as it does exist. However, the frequent use of RREQ/RREP may result in increased latency and energy consumption due to its flooding nature. To minimize the use of RREQ/RREP, we employ the following two location-based optimization techniques.

\subsection{M-Geocast Optimizations}

Geographic Void Prediction: To minimize the use of RREQ/RREP, we should avoid encountering a geographic hole whenever possible. From our simulation studies we found that if the direction pointed by the geometric routing is the opposite direction of the recent path history, there is a high chance of a geographic hole since the location updates from the master sink usually takes the shortest path. If such condition is met, M-Geocast predicts that the geometric routing may lead to a geographic hole and uses the path history instead. This is implemented by computing cosine $\theta$ between the direction of greedy forwarding and the direction pointed by the path history. If the value of cosine $\theta$ is greater than $0, \mathrm{M}$-Geocast adopts the path history. Otherwise, $\mathrm{M}$ Geocast uses the greedy forwarding. To prevent a loop in the routing path, M-Geocast does not use greedy forwarding once path history is selected. As shown in Section IV, this can effectively reduce the number of geographic holes.

Path History Projection: The second option in minimizing the use of RREQ/RREP is to avoid a history hole. However, it is physically impossible to avoid a link failure if the next hop has disappeared due to node movement. Yet, we can still approximate the path history on a link failure. By using the location information of its neighbors, the forwarding node can send messages to a neighbor node which is located in the same direction as pointed by the previous path history. This is also implemented by selecting a neighbor node among its neighboring nodes that has the maximum cosine $\theta$ from the direction pointed by the path history. To prevent a routing loop, node $\mathrm{S}$ must be removed from the new path history. Note that both of these optimizations are possible due to location information of neighbor nodes, which are available only in geometric routing schemes. 


\section{Simulation}

\subsection{Simulation Methodology}

We have implemented all the routing functions of M-Geocast in the ns-2 simulator [12]. We generate 200-node sensor field by randomly placing the nodes in a $1500 \times$ $1500 \mathrm{~m}^{2}$. Each node has a radio range of $250 \mathrm{~m}$. Unless otherwise mentioned, all sources are randomly selected from the sensor field following the random sources model [1] while sinks are uniformly scattered across the field. Each source generates one event per second and each event is modeled as a 64-byte packet. All the events are reported to all the sinks in the field. We use the random waypoint model [3] as a mobility model. The maximum pause time is 30 seconds. Each simulation run lasts for 100 seconds.

We use three metrics: average message latency, average dissipated energy, and delivery success ratio. Average message latency measures the average one-way communication latency between a source and a sink. Average dissipated energy measures the ratio of total dissipated energy per node to the number of distinct events seen by sinks. Delivery success ratio is the ratio of the number of distinct events successfully received to the total number of events originally reported. Similar metrics were used in early works $[2,5,8]$. For each metric, we vary the number of sources, the number of sinks, node speed, and node density.

All the metrics of M-Geocast are compared against a baseline geographic greedy forwarding (Geocast) and Active On-demand Distance Vector routing (AODV) [8]. Geocast is used to evaluate the impact of master sink in M-Geocast and AODV is one of the most popular and effective MANET routing protocol. We use the same energy model and MAC protocol as adopted in their ns-2 simulators. We use 1.6Mbps 802.11 $\mathrm{DCF}$ as the underlying MAC protocol. The idle-time power dissipation is $35 \mathrm{~mW}$, reception power dissipation is $395 \mathrm{~mW}$, and transmission power dissipation is $660 \mathrm{~mW}$.

\subsection{Simulation Results}

Varying the number of sinks: Figure 3 shows the results by varying the number of sinks from 1 to 16 . Five sources were used for the simulations. Figure 3(a) shows the average message latency. AODV's latency is substantially higher than those of geometric schemes when the number of sinks is greater than 4. This is because RREQ flooding required by AODV generates significant traffic, and the additional contention induced by such traffic increases delay significantly. In Geocast the situation is less severe but its average message delay start to increase as the number of sinks grows since each sink generates location updates every 3 seconds during its movement. However, the delay remains constant in M-Geocast for all the cases simulated, which is noticeably faster than that of Geocast. Figure 3(b) shows the average dissipated energy. As the number of sinks grows, the gap between M-Geocast and other schemes start to grow substantially. For the 16 sink case, M-Geocast consumes only $41 \%$ of AODV's and $51 \%$ of Geocast's average energy consumption respectively. MGeocast's energy savings are due to the fact that it requires location update from a single master sink while AODV and Geocast incur substantial overhead due to RREQ 
flooding and location broadcasts from multiple sinks respectively. Finally, Figure 3(c) shows the delivery success ratio. For all the cases simulated M-Geocast successfully delivers more than $99 \%$ of all the events. Although Geocast shows comparable delivery ratios but its ratio drops to $94 \%$ with 16 sinks. In contrast, the delivery success ratio of AODV suddenly drops to $12 \%$ as the number of sinks increases from 4 to 8 . This is because AODV incurs RREQ path setup for each sink.

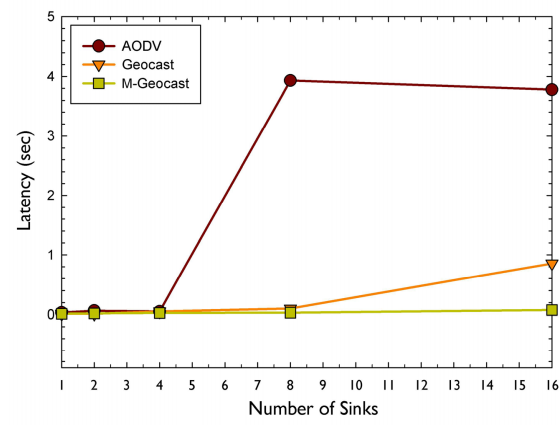

(a) Average message delay

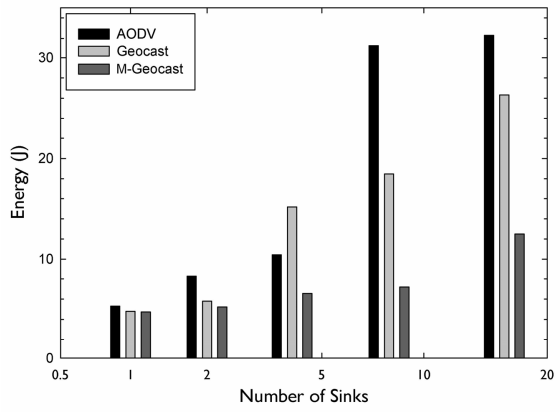

(b) Average dissipated energy

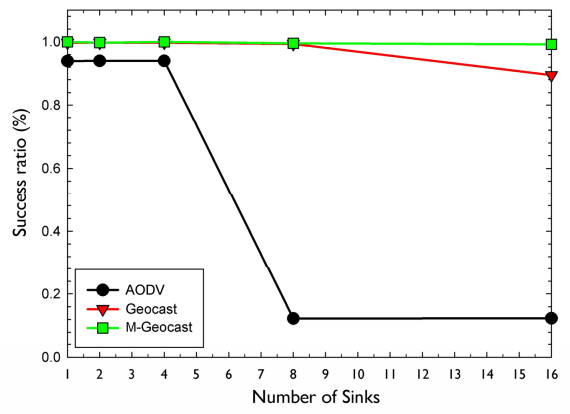

(c) Delivery success ratio

Fig. 3. Varying the numbers of sinks

Varying the number of sources: Our second set of simulations evaluates the performance of M-Geocast by varying the number of sources from 1 to 16 , which is shown in Figure 4. Two sinks are assumed for the simulations. As shown in the figure the latency of AODV increases substantially as we increase the number of sources because AODV requires RREQ flooding for each source. The numbers of both Geocast and M-Geocast are comparable and substantially smaller than that of AODV. In addition, the average dissipated energy of M-Geocast is smaller than Geocast and AODV especially when there are a large number of sources. This is because the protocol overhead incurred by AODV and Geocast steadily increases as the number of sources grows compared to M-Geocast. With 16 sources, M-Geocast's energy consumption is less than $33 \%$ of AODV and $66 \%$ of Geocast. Finally, M-Geocast successfully delivers almost all the events to the sinks while Geocast's delivery ratio is 


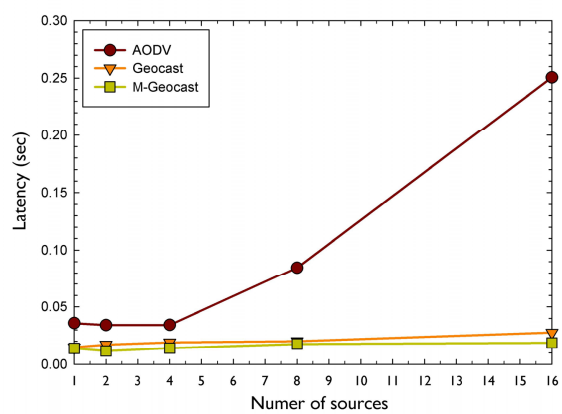

(a) Average message delay

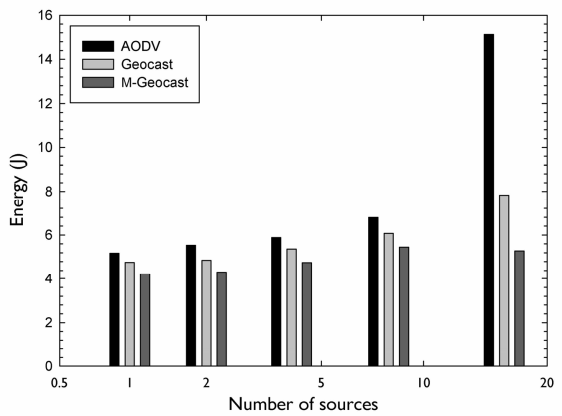

(b) Average dissipated energy

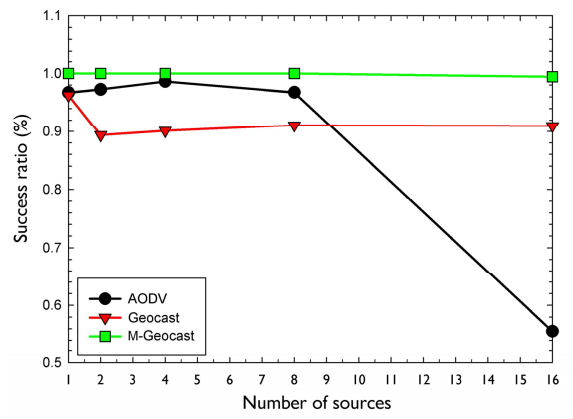

(c) Delivery success ratio

Fig. 4. Varying the numbers of sources

around $91 \%$ for multiple sources and AODV's delivery ratio suddenly drops down to below $60 \%$ as the number of sources reaches 16 .

Varying the node speed: We next evaluate the impact of node speed on the performance of M-Geocast. Five sources and four sinks are used in each simulation run. Figure 5 compares the three metrics by varying the average speed of each node from 0 to $20 \mathrm{~m} / \mathrm{s}$. As the node speed increases, both the delay of both Geocast and M-Geocast remains stable. This is because geometric routing does not incur additional overhead regardless of its speed as long as the location information of the destination remains valid. Surprisingly, Geocast's latency actually decreases as the node speed increases. This is due to the fact that Geocast cannot deliver some of its messages for medium to high speed movement as illustrated in Figure 5(c). Compared to the geometric schemes, the average message latency of AODV substantially increases as the node speed increases. As the node speed increases, the existing route entries cached by AODV become no longer valid. This requires AODV to flood RREQ for each message generated. As a result, the average message delay of AODV linearly increases. The latency of M-Geocast remains below 38ms while the latency of Geocast varies for different node speed due to its unstable message delivery. For all the cases simulated M-Geocast's average dissipated energy remains relatively constant, around 6.5J while the energy consumption of AODV steadily increases. As we expect from the 


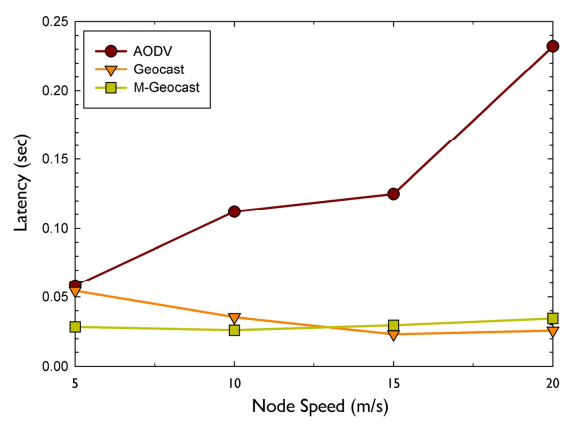

(a) Average message delay

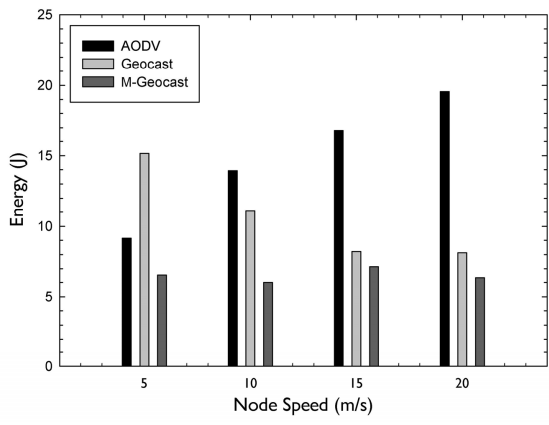

(b) Average dissipated energy

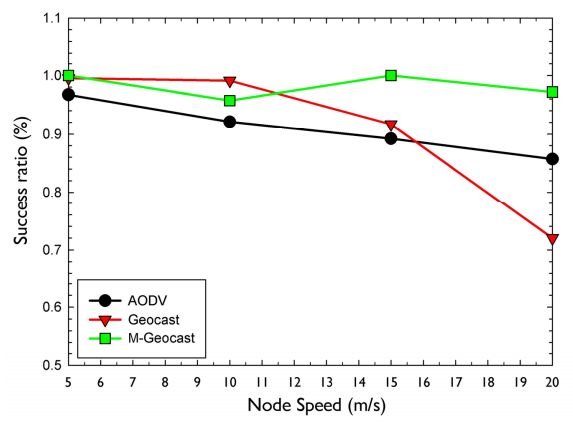

(c) Delivery success ratio

Fig. 5. Varying the node speed

low delay and the low energy consumption of M-Geocast, M-Geocast consistently delivers more than $96 \%$ of all the events for all the cases, whereas Geocast's success ratio drops down to $72 \%$ for nodes with high speed, which is even lower than that of AODV. Yet, AODV's success ratio steadily decreases to $86 \%$ when the sink speed reaches $20 \mathrm{~m} / \mathrm{s}$.

\subsection{Detailed Evaluation of M-Geocast Under Routing Holes}

In this section, we evaluate the impact of routing holes on the performance of MGeocast by varying the node density in the sensor field. 16 sources and a single sink are used in each simulation run. Three versions of M-Geocast are tested as follows.

- GHR (Geocast $\rightarrow$ History $\rightarrow$ RREQ/RREP): In this scheme, a source node first uses geographic greedy forwarding. On a geographic hole, it rediscovers a second path using path history. If both fail, the scheme employs on-demand route discovery using RREQ/RREP.

- HGR (History $\rightarrow$ Geocast $\rightarrow$ RREQ/RREP): In this scheme, a source node first uses path history to find a path to the destination. If this leads to a broken link, the scheme tries geographic greedy forwarding. If both fail, the scheme uses on-demand route discovery. 
- MG-Opt (HGR with two optimizations): The scheme is essentially same as HGR except that it uses two optimization techniques, geographic void prediction and path history projection to minimize the use of RREQ/RREP.

Figure 6 compares the three metrics by varying the average number of neighbors per node from 8 to 30 . When the node density is below 10, the latency of both GHR and HGR suddenly increases. This is due to the increased number of geographic holes due to the low node density. The latency of HGR is slightly higher than GHR because we only show the average delay of messages that are successfully delivered. In fact, the delivery success ratio of HGR is higher as shown in Figure 6(c). In addition, the latency of HGR is also higher than that of GHR for high density case. However, in this case, the delivery success ratio of GHR is improved as the number of routing hole diminishes as shown in Figure 6(d). Thus, for a low density case HGR generally outperforms GHR while for a high density case both schemes show comparable performance. In contrast, the average message latency of MG-opt is lower than both schemes and remains stable throughout all simulation cases. Figure 6(b) shows the per-node average energy dissipation of the three M-Geocast versions. All three schemes show comparable energy consumption. GHR shows slightly a lower energy dissipation but at the cost of low delivery success ratios. Figure 6(c) shows the delivery success ratios of three M-Geocast schemes. As we expect from the low delay and the low number of

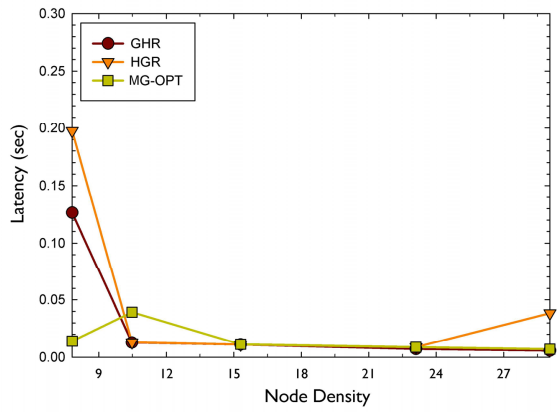

(a) Average message delay

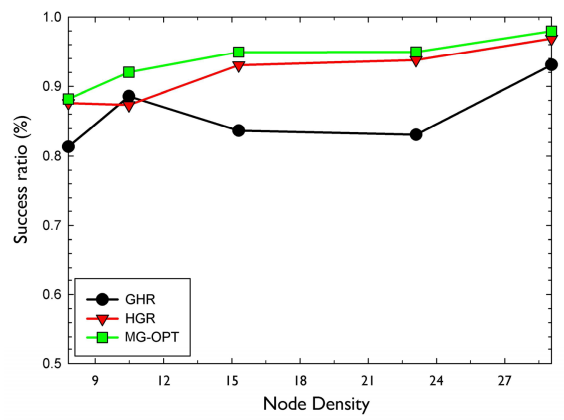

(c) Delivery success ratio

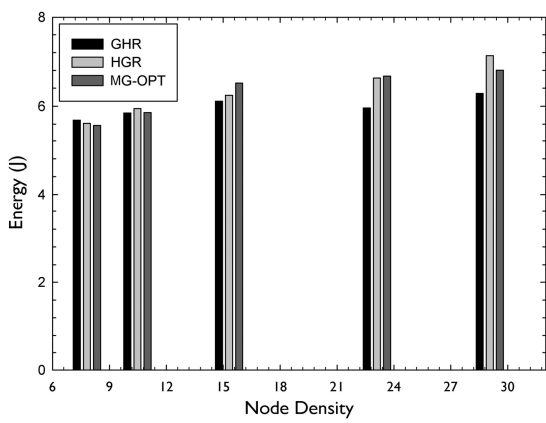

(b) Average dissipated energy

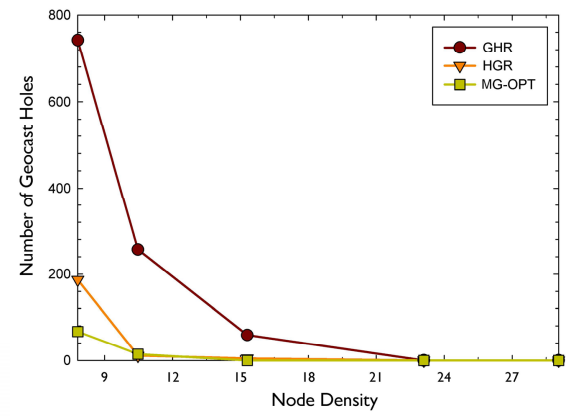

(d) Number of geographic holes

Fig. 6. Impact of routing holes by varying the node density 
routing holes, MG-opt consistently delivers more than $89 \%$ of all the events for all the cases, which is higher than HGR and GHR. In fact, GHR's success ratio drops down to $81 \%$ for low to medium density. This suggests that the two optimizations can effectively improve the performance of M-Geocast in terms of both average message delay and delivery success ratio. Figure 6(d) shows the number of geographic holes encountered during simulations by varying the node density. As shown in the figure, HGR can effectively reduce the number of geographic routing holes compared to GHR by trying history path first and MG-opt can further reduce the number by using the geographic void prediction and path history projection.

\section{Conclusion}

This paper proposes a new geometric routing protocol called M-Geocast that is specifically designed to support mobile sensor networks. M-Geocast provides scalable and energy efficient routing and location service that targets many-to-one traffic pattern of wireless sensor network and achieves significant performance and energy improvement over existing MANET routing protocols. Our detailed experimentation results on the ns-2 platform confirm that M-Geocast can significantly save energy while it can also reduce both the message delay and the message delivery failures compared to AODV and a plain geometric routing scheme. We also address the issue of routing holes, which has been the key focus of existing geometric routing schemes. By using the path history of location updates and also taking simple yet effective location-based optimization techniques, we can substantially reduce the number of geographic holes. As a result M-Geocast can effectively reduce both the energy consumption and average message delay compared to existing geometric schemes. This suggests that $\mathrm{M}$-Geocast can be a scalable yet robust routing solution for mobile sensor networks when implemented with GPS-enabled mobile sensor nodes.

\section{References}

1. Al-Karaki, J.N., Kamal, A.E.: Routing Techniques in Wireless Sensor Networks: A Survey. Wireless Communications 11(6), 6-28 (2004)

2. Choi, L., Choi, K., Kim, J., Park, B.J.: Virtual Sink Rotation: Low-Energy Scalable Routing Protocol for Ubiquitous Sensor Networks. In: Proceedings of the 1st International Workshop on RFID and Ubiquitous Sensor Networks (USN 2005), Japan (December 2005)

3. Johnson, D.B., Maltz, D.A.: Dynamic Source Routing in Ad Hoc Wireless Networks. Mobile Computing, 153-181 (1996)

4. Juang, P., Oki, H., Wang, Y., Martonosi, M., Peh, L.S., Rubenstein, D.: Energy-efficient computing for wildlife tracking: design tradeoffs and early experiences with zebranet. In: Proceedings of the ACM 10th international conference on architectural support for programming languages and operating systems (ASPLOS-X), pp. 96-107 (2002)

5. Karp, B., Kung, H.T.: GPSR: Greedy Perimeter Stateless Routing for Wireless Networks. In: Proceedings of Mobicom 2000 (2000)

6. Li, J., et al.: A Scalable Location Service for Geographic Ad Hoc Routing. In: Proceedings of Mobicom 2000, pp. 120-130 (2000) 
7. Mauve, M., Widmer, J.: A Survey on Position-Based Routing in Mobile Ad Hoc Networks. IEEE Network (November/December 2001)

8. Perkins, C., Royer, E.: Ad-hoc On-Demand Distance Vector Routing. In: Proceedings of the 2nd IEEE Workshop on Mobile Computing Systems and Applications (1999)

9. Poduri, S., Sukhatme, G.S.: Constrained Coverage for Mobile Sensor Networks. In: Proceedings of IEEE International Conference on Robotics and Automation (May 2004)

10. Stojmenovic, I.: Home Agent Based Location Update and Destination Search Schemes in Ad Hoc Wireless Networks. Technical Report TR-99-10, University of Ottawa (September 1999)

11. Tilak, S., Kolar, V., Abu-Ghazaleh, N.B., Kang, K.D.: Dynamic Localization Control for Mobile Sensor Networks. In: IEEE International Workshop on Strategies for Energy Efficiency in Ad Hoc and Sensor Networks (April 2005)

12. USC Information Science Institute, The Network Simulator ns-2 Documentation (2002), http: //www.isi.edu/nsnam/ns/ 